\title{
Représentations littéraires des paysages miniers en Nouvelle-Calédonie : regards coloniaux et vécus kanak
}

Literaty representations of mining landscapes in New Caledonia : colonial perspective and Kanak experience

\section{Eddy Banaré}

\section{(c) OpenEdition \\ Journals}

Édition électronique

URL : http://journals.openedition.org/jso/7080

DOI : $10.4000 /$ jso. 7080

ISSN : $1760-7256$

\section{Éditeur}

Société des océanistes

Édition imprimée

Date de publication : 15 décembre 2014

Pagination : 151-164

ISBN : 978-2-85430-118-2

ISSN : 0300-953x

\section{Référence électronique}

Eddy Banaré, «Représentations littéraires des paysages miniers en Nouvelle-Calédonie : regards coloniaux et vécus kanak », Journal de la Société des Océanistes [En ligne], 138-139 | 2014, mis en ligne le 15 décembre 2017, consulté le 20 avril 2019. URL : http://journals.openedition.org/jso/7080 ; DOI : $10.4000 /$ jso. 7080 


\section{Représentations littéraires des paysages miniers en Nouvelle-Calédonie : regards coloniaux et vécus kanak}

par

Eddy BANARÉ*

\section{RÉSUMÉ}

Montagnes ravinées, minéraliers et usines sont, depuis la fin du XIX siècle, autant de motifs décrits pour évoquer la Nouvelle-Calédonie. Des récits ont aussi inscrit l'exploitation minière dans l'imaginaire de Nouvelle-Calédonie. L'élaboration des discours sur la mine en Nouvelle-Calédonie a relevé, dès les années 1880, d'une question de maintien de la domination coloniale. Il s'agissait alors d'améliorer l'image de la Nouvelle-Calédonie en tant que colonie française. Différents types de discours ont ainsi participé à la construction d'un imaginaire de la mine en NouvelleCalédonie. La singularité de ces discours littéraires est de rendre visible l'irruption de la mine dans l'imaginaire kanak dont l'interprétation est essentielle pour saisir les enjeux contemporains locaux.

MoTS-CLÉS : littérature, colonisation, mine, paysages, Kanak

\section{La mine entre politique et géopoétique}

L'activité minière a connu des mises en récits qui, du fait des différences d'époque et d'origine de leurs auteurs, peuvent être reçues comme autant de témoins de l'élaboration d' "identités narratives" «issue(s) de la rectification sans fin d'un récit antérieur par un récit ultérieur, et de la chaîne de refigurations qui en résulte" (Ricœur, 1985 : 446). La mine est un carrefour

\begin{abstract}
Since the end of the $19^{\text {th }}$ century, dramatic mountains, ore carriers and factories have been among the images that represent New Caledonia. In addition to the landscape, various narratives and stories have etched the mining industry into the imagery of New Caledonia. From the 1880s, the development of discourse on mining in New Caledonia responded to the need to preserve colonial domination. In order to maintain this domination, the image of New Caledonia as a French colony needed to be improved. Different types of discourse contributed towards constructing a picture of mining in New Caledonia. Literary discourse has a unique ability to unfold the emergence of mining in the Kanak imagination, the interpretation of which is essential to the understanding of contemporary issues in the area.
\end{abstract}

KeYwords: literature, colonization, mining, landscapes, Kanak

anthropologique, elle sollicite plusieurs de ces " identités narratives » qui constituent la NouvelleCalédonie contemporaine. L'exploitation minière est en effet le résultat des ambitions coloniales du Second Empire. Condamnés et colons s'y sont trouvés réunis, des clans kanak ont été déplacés de force, des champs détruits pour permettre l'extraction du minerais. Et, de de la fin du XIX ${ }^{e}$ siècle aux années 1930, des travailleurs asiatiques y ont été engagés sous contrat. Pour ces 
derniers, on trouve déjà une production littéraire témoignant de ce vécu minier (Do, 2008) avec les romans Chân Dăng et Fils de Chân Dăng de Jean Vanmaï publiés en 1980 et 1983 . Du point de vue documentaire, la biographie (Pitoiset et Wéry, 2009) d'André Dang, fils d'engagés indochinois devenu en 2000 directeur de la SMSP (Société minière du Sud Pacifique), écrite par les journalistes Anne Pitoiset et Claudine Wéri, offre un éclairage sur l'histoire politique récente. Lié à Jean-Marie Tjibaou, André Dang est en effet celui qui a permis l'entrée des Kanak dans l'économie du nickel (Pitoiset et Wéry, 2009).

La liberté de la recherche littéraire est de pouvoir se situer aux points extrêmes afin de confronter les points de vue en prenant le parti, par exemple, de considérer que la vision des paysages miniers d'une poétesse kanak comme Déwé Görödé pendant les "Événements " (situation insurrectionnelle des années 1984-1988) pourrait être une réponse donnée à un chroniqueur colonial de la fin du XIX ${ }^{\mathrm{e}}$ siècle. Il en est de même pour la pièce du dramaturge kanak Pierre Gope, qui peut être reçue comme un témoignage sur le devenir politique. Kanak et européennes, ces identités narratives sont historiquement antagonistes en Nouvelle-Calédonie et travaillées par un legs colonial. Le devenir politique initié par l'accord de Nouméa depuis 1998 - accord politique marquant l'entrée dans une période de "décolonisation négociée " - suggère que les sciences humaines peuvent approcher l'activité minière afin de formuler des interrogations à la fois sociales, culturelles, environnementales et économiques, nécessaires au rééquilibrage géographique et socio-ethnique voulu par cet accord. Il s'agit de s'insérer dans cette " chaîne de refigurations " (ibid.), afin d'identifier les termes d'un dialogue autour du premier secteur économique de la Nouvelle-Calédonie et d'un processus politique au coeur duquel se trouve la reconnaissance de l'identité kanak. Avant la poésie et le théâtre, l'activité minière a été prise en charge par le monde kanak à travers le mémoire d'étude d'Apollinaire Anova, séminariste originaire de Moindou, décédé en 1966. Le document intitulé Histoire et psychologie des Mélanésiens ${ }^{1}$ d'abord de manière souterraine dans le milieu étudiant kanak (Chappell, 2013) avant que des extraits en soient publiés en 1969 dans le Journal de la Société des Océanistes sous le titre "Deux exemples de réflexions mélanésiennes" (Anova-Ataba, 1969). Au croisement de l'anthropologie, de la sociologie et de l'économie, Anova y développe une réflexion sur la Nouvelle-Calédonie de son époque et y propose des solutions intellectuelles nécessaires à la participation des Kanak dans le tissu économique. Parmi ces solutions figure la réhabilitation du chef Ataï, à l'origine de la révolte de 1878 (Mokaddem, 2014). S'il ne s'agit pas de fiction ou de poésie, ce texte constitue le fondement du nationalisme kanak, et sa dimension littéraire réside dans la prise en charge de la figure d'Ataï, nourrie de récits oraux. Anova propose une reconfiguration de la révolte de 1878 où, selon Hamid Mokaddem, «l'écriture est une fiction historique qui fait dialoguer les parties en présence" (Mokaddem, 2014 :7). L'influence d'Anova a également été revendiquée par JeanMarie Tjibaou, Nidoish Naisseline ou encore Elie Poigoune (Chappell, 2013). On peut considérer que la filiation avec la création littéraire qui a accompagné le nationalisme s'établit à partir de 1985, avec la publication du recueil Sous les cendres des conques par Déwé Görödé. Un détour par la création littéraire pourrait aider à penser la mise en œuvre des politiques (sociales, économiques et écologiques) à partir des regards posés sur les paysages (Roe, 1994). En effet, Fredric Jameson a décrit « la littérature comme acte socialement symbolique "(Jameson, 2012), partant du principe que "l'acte littéraire ou esthétique entretient toujours un rapport actif au Réel " (Jameson, 2012:100) et qu'il est, en cela, porteur d'interrogations et d'idéologies élaborées en regard de la politique. Relire ce qui a été dit sur les paysages, interpréter ce qui en a été vu, c'est " ouvrir les yeux sur l'histoire», au sens où l'entend Georges Didi-Huberman, c'est-à-dire, " commencer par temporaliser les images qui nous en restent" (Didi-Huberman, 2010: 27). Une partie de ces images fixées et données par la littérature nous permet de réinventer indéfiniment un passé, mais aussi une manière de distribuer des rôles dans le présent de la société pluriethnique de la Nouvelle-Calédonie. C'est en ce sens que la signature de l'accord de Nouméa en 1998 impose un passage par la politique et un rapport radicalement renouvelé à l'histoire en ce qu'elle est conçue comme, précise encore Jameson,

« ce qui fait mal, ce qui refuse le désir, ce qui impose des limites inexorables à la praxis individuelle comme collective, ce qui par ses "ruses", renverse, de façon horrible ou ironique, les intentions affichées » (Jameson, 2012 : 127).

Ainsi, «l'acte littéraire» doit-il être pris comme une tentative, par le langage, de dépasser ces "limites" qui, dans le cas de la NouvelleCalédonie, sont celles de la colonisation. Ce qui a été "renversé " avec l'exploitation minière ce sont, du point de vue kanak, des manières de

1. Le texte sera d'ailleurs réédité pendant les Événements en 1985 sous le titre D'Ataï à l'indépendance aux éditions Édipop à Nouméa, la même année que le recueil Sous les cendres des conques de Déwé Görödé. Une seconde édition établie par Bernard Gasser et Hamid Mokaddem sera publiée en 2005 sous le titre Calédonie d'hier, Calédonie d'aujourd'hui, Calédonie de demain aux éditions Expression. 
vivre de la terre et d'y circuler. Le préambule de l'accord de Nouméa appelle également à des approches plus sensibles des lieux et des mémoires susceptibles de rejaillir sur les décisions politiques. C'est en ce sens que la recherche littéraire peut être considérée comme un des moyens de saisir la complexité des expériences du nickel propres à la Nouvelle-Calédonie. On retiendra les commentaires de François Garde qui observe que la force de ce préambule est :

" [...] qu'il propose une histoire qui n’a de vertu que si les Calédoniens se reconnaissent en elle. Il ne prétend pas au monopole de la pensée historique, mais par ses contradictions et ses silences, appelle la critique et la recherche. » $(2005: 811)$

La littérature permettrait de restituer des expériences structurantes de l'activité minière en tant que lieu incontournable d'une "pensée historique ". Les manières de dire le passé déterminent le vécu du présent. Il est évident que les politiques sont différentes selon que l'on se considère descendant de pionnier ou victime du colonialisme. On n'en saisit que mieux cette autre observation de Jameson selon laquelle

« la distinction opératoire que l'on établit par commodité entre des textes culturels, sociaux et politiques et d'autres qui ne le sont pas, est pire qu'une erreur [...] elle confirme le décalage entre l'histoire ou la société et "l'individuel" [...] qui mutile notre existence de sujets individuels, paralyse notre pensée du temps et du changement autant qu'il nous aliène notre propre parole. " (Jameson, 2012:19)

D'où la nécessité d'une approche de la littérature comme matériau mémoriel. Le premier alinéa du préambule de l'accord de Nouméa institue l'idée d'injustices coloniales ${ }^{2}$, en appelant à "faire mémoire des moments difficiles" (JORF 121, 27 mai 1998). Le texte insiste sur le fait que "l'identité kanak était fondée sur un rapport à la terre " (ibid.), et surtout sur ce que «les noms que la tradition donnait à chaque élément du paysage, les tabous marquant certains d'entre eux, les chemins coutumiers structuraient l'espace et les échanges » (ibid.).

Il y a, dans l'exploitation coloniale, un rapport qui s'instaure avec les paysages; un moment où le colon se proclame pionnier et dit : « il n'y a rien ici " (Petit, 2010) et considère que les ressources sont librement accessibles; c'est le moment de la force (force qui s'exerce aussi via des controverses juridiques; $c f$. Merle, 1998 sur ce point). Vient ensuite le moment de l'appropriation et de la célébration patriotique: les stations minières, le ballet des minéraliers et les hauts-fourneaux signalent, selon une certaine propagande, l'excellence coloniale. Enfin, le troisième moment, plus intime, plus "romantique" est celui où le paysage signifie à la fois sueur, sang et sacrifices, le moment où les paysages finissent par rentrer dans la composition même de la chair, où ils constituent la mémoire d'un passé idéalisé, voire mythifié. Pour que s'opèrent ces transformations, la mine a dû se constituer comme objet et représentation chez les colons de la Nouvelle-Calédonie. La littérature coloniale permet de saisir la nature de rapports aux lieux et aux populations kanak déplacées de force. C'est par la presse que les paysages miniers ont intégré les imaginaires à partir de 1880 .

Comme l'ont montré les travaux de Yann Bencivengo et de Catherine Buttet, l'activité minière était entourée d'un voile opaque jeté tant par les banques (Buttet, 2000) que par l'administration coloniale (Bencivengo, 2008). Il faut ajouter que la presse contrôlée par le monde des affaires participait à une intense propagande afin de calmer une colonie pénale sous tension (Coquilhat, 1987). La littérature ainsi publiée n'échappait pas à ce rôle; elle imposait un langage et une représentation du monde du nickel. Il existait néanmoins une presse de contestation, certes minoritaire, qui permet de saisir les antagonismes en jeux à la fin du $\mathrm{XIX}^{\mathrm{e}}$ siècle en Nouvelle-Calédonie. On peut dire que l'émergence de la littérature kanak à partir de la publication du recueil de Déwé Görödé en 1985 relève d'un effort de se saisir de la complexité politique dont relève le nationalisme kanak et les enjeux économiques miniers qui, comme on le voit avec le drame de Pierre Gope, apparaissent également opaques et redoutablement complexes.

\section{Quatre moments dans la mise en récit de la mine en Nouvelle-Calédonie}

Notre choix s'est porté sur quatre textes qui témoignent de ces mémoires en jeu et symbolisent les écarts fondateurs de la NouvelleCalédonie. D'abord sur le plan historique: nous avons compilé la chronique que le député Francisque Ordinaire (1844-1896) rédige lors de sa visite en Nouvelle-Calédonie en 1889. Après avoir lu une critique dont cette chronique fait l'objet en 1891, nous la mettrons en lien

2. Voici l'intégralité de l'alinéa 1 du préambule de l'accord de Nouméa : " Lorsque la France prend possession de la Grande Terre, que James Cook avait dénommée "Nouvelle-Calédonie", le 24 septembre 1853, elle s'approprie un territoire selon les conditions du droit international alors reconnu par les nations d'Europe et d'Amérique, elle n'établit pas des relations de droit avec la population autochtone. Les traités passés, au cours de l'année 1854 et les années suivantes, avec les autorités coutumières, ne constituent pas des accords équilibrés mais, de fait, des actes unilatéraux. " (JORF 121, 27 mai 1998) 
avec la nouvelle "Sauvages et Civilisés " écrite par Georges Baudoux (1870-1949) en 1915. Ordinaire pose le regard d'un visiteur - d'un homme politique en campagne - sur les colonies. Baudoux, au contraire, est un "enfant du pays " (Gasser, Sollier, 1996), self-made man respecté et admiré : son enracinement est différent. Pour l'époque contemporaine, les extraits du recueil Sous les cendres des conques que Déwé Görodé publie en 1985 et le drame de Pierre Gope intitulé Le dernier crépuscule témoignent des transformations de l'expérience kanak face aux mutations induites par l'implication dans l'exploitation minière. Nous nous intéresserons d'abord à la genèse et aux grandes transitions de cette géopoétique coloniale des paysages miniers et à leur influence dans l'élaboration de la mémoire collective, pour nous intéresser ensuite aux contenus des revendications indépendantistes kanak, incluant les motifs du rééquilibrage et de la préservation de l'environnement. Notre point de départ sera la chronique de Francisque Ordinaire, ancien député en visite en NouvelleCalédonie en 1889 au centre minier de Thio, sur la côte Est. Ce centre, alors amené à être l'un des plus importants de la Nouvelle-Calédonie, est encore en activité (Le Meur, 2009). C'est l'un des premiers témoignages sur les paysages après la fondation de la SLN en 1880 (Bencivengo, 1999). Ordinaire est en campagne, il est connu pour s'être engagé dans les troupes de Garibaldi en 1866. Il est socialiste et membre de l'Union républicaine, et son rapport aux paysages est marqué par une volonté de témoigner d'une connaissance de l'économie coloniale. Il mobilise longuement des chiffres, des noms, et des informations historiographiques, semblant un temps "oublier" la réalité des paysages miniers. Éloigné des affaires politiques depuis sa défaite aux législatives de 1877 , Ordinaire ne semble avoir eu aucun contact préalable avec les communards emprisonnés en NouvelleCalédonie qui auraient pu lui livrer quelques informations de première main. Nous verrons même que son point de vue sera critiqué par deux d'entre eux.

Dans la nouvelle "Sauvages et Civilisés" écrite en 1915, Georges Baudoux montre que l'attachement la terre (à travers sa nature et ses paysages) est incompatible avec l'exploitation minière qui faisait la fierté coloniale. Continuer à exploiter le minerai, c'est perdre un paradis ; celui, intime, du pays d'enfance et des premiers émerveillements. Fils d'un fonctionnaire de l'administration pénitentiaire arrivé en 1874, Baudoux a fréquenté la plupart des lieux "stratégiques » : le bagne où il a été éduqué, la brousse où il été stockman. Il a aussi fréquenté le monde kanak. La mine est, pour lui, un paysage secondaire : il a vécu les débuts de la SLN et vu les " prospects $^{3}$ » et décapages (qui consistent en des abrasements de surfaces afin de révéler l'éventuel filon). Les flancs des montagnes sont marqués de sillons rougeâtres (pareils à des balafres). La popularité qu'il connait en tant qu'auteur est fondée sur cette expérience singulière de la colonie. Capable de retranscrire des contes kanak (écoutés lors de ses veillées en tant que stockman parmi ses employés kanak) et de faire fortune grâce à l'extraction du cobalt à Koné à partir de 1895 (Banaré, 2012 : 249-258), année où il entame également sa carrière d'auteur de feuilletons, il est l'archétype de la débrouillardise et de la réussite coloniale. Mais nous verrons qu'il est également porteur d'une ambivalence fondatrice.

Enfin, la dernière partie de l'article s'intéressera aux contestations et aux interrogations contenues dans la prise de parole contemporaine des auteurs kanak (Mokaddem, 2007). Les textes de Déwé Görödé et de Pierre Gope marquent une véritable césure dans notre corpus. Pour commencer, ils viennent du monde kanak qui, jusqu'aux années $\mathrm{du}$ «boom» du nickel (Bensa et Freyss, 1994), était fortement marginalisé et réduit au silence par l'activité minière. Et surtout, ils encadrent trois séquences historiques déterminantes: la période post-indigénat jusqu'au "boom " (1946-1972), les «Événements " (1984-1988) pour Görödé, et l'accord de Nouméa en 1998 pour Pierre Gope.

Originaire de Pwârâiriwâ (Ponérihouen), Déwé Görödé a suivi une formation universitaire en littérature en France. De retour en 1974, elle se fait connaitre en tant qu'activiste au sein des mouvements Foulards rouges et Groupe 1878. Elle a également participé à la création du Palika (Parti de libération kanak) en 1976 qui est une des composantes du FLNKS (Front de libération kanak et socialiste) fondé en 1984. Enseignante pendant les "Événements " au sein de l'EPк (École Populaire Kanak) de sa région d'origine, elle publie le recueil poétique Sous les cendres des conques en 1985. Ce volume reprend également des poèmes composés pendant ses années passées militantes. Elle devient membre du gouvernement de la Nouvelle-Calédonie dès 1999, poste qu'elle occupe actuellement en tant que représentante du PaLiKa (Parti de libération kanak). Sous les cendres des conques contient une section consacrée à l'exploitation minière intitulée "Terre Nickel ». L'association des deux termes signale ici l'antinomie fondatrice: la "Terre " évoque le sacré et la maternité, alors que le nom "Nickel » semble s'être ici substitué à l'adjectif "sacrée» pour évoquer l'industrie 
déshumanisée et la froideur du métal. On peut comprendre les poèmes qui composent cette section comme l'exploration douloureuse d'une blessure historique. Ces poèmes, composés entre 1970 et 1985 , disent tous la marginalisation du monde kanak par rapport au monde minier. L'exploitation minière est perçue comme une activité coloniale.

Pierre Gope est né à Maré en 1966. Il entame une carrière d'animateur culturel en 1990, ce qui l'amène à traverser l'ensemble de la GrandeTerre pour un travail de collecte d'informations. En 1992, il met en scène sa première pièce et fonde sa troupe, Cebue. Il s'impose comme un observateur attentif et critique d'une société kanak en profonde transformation. Le dernier crépuscule, créé en 2000, donne à voir les difficultés qui existent encore. Surtout, l'œuvre interroge les défis à relever pour le monde kanak désormais activement impliqué dans les activités minières. Fondé sur le conflit des générations, $L e$ dernier crépuscule met en scène la confrontation entre les anciens et un jeune diplômé kanak qui tente de les convaincre de céder les terres ancestrales à une compagnie minière. Ce drame interroge les modalités d'une transformation sociale et celles de l'exercice des nouveaux pouvoirs de décision acquis de haute lutte.

Ces deux textes kanak s'inscrivent dans le sillage de deux moments fondateurs : le recueil Sous les cendres des conques est l'œuvre des "Événements " (1984-1988). À bien des égards, il est considéré comme le manifeste poétique du mouvement indépendantiste kanak et l'œuvre fondatrice de la littérature kanak moderne. Le dernier crépuscule de Pierre Gope est joué pour la première fois en 2000, deux ans après la signature des accords fondateurs : celui de Bercy, signé en février, qui acte l'entrée des kanak dans l'activité minière à travers la création d'une usine dans le nord de la Grande Terre, et l'accord de Nouméa, signé le 5 mai qui porte sur la mise en ouvre d'une décolonisation fondée sur le rééquilibrage (Gagné, Salaün 2013). Ces textes scandent l'activité minière: les premiers ont permis d'imaginer une prospérité à venir, d'autres ont fait naître une communauté. C'est sur ce point que l'expression littéraire est précieuse dans la mesure où, par la poétique et la fiction, un pays s'invente et s'imagine (Anderson, 2006).

De même, nous dit Jacques Rancière :

"la littérature est "sociale", elle est l'expression d'une société en ne s'occupant que d'elle-même, c'est-à-dire de la manière dont les mots contiennent un monde. " (Rancière, 2010 : 45)

Ce que la littérature rend visible en matière minière, est la nature des liens entre la politique, le monde des affaires et les communautés qui étaient déplacées et/ou amenées à travailler dans les mines. Un processus de familiarisation et de transformation est également mis en évidence à travers le langage qui est restitué par la littérature. La mine est un nouveau lieu qui se constitue avec ses codes particuliers. Ces textes permettent d'approcher, du moins en partie, ce que les paysages miniers de la Nouvelle-Calédonie ont pu dire et permettre d'imaginer comme futurs - ceci, en parallèle des considérations économiques et des négociations politiques.

\section{Contradictions coloniales et mémoires minières}

Comment, aux débuts de l'exploitation minière en Nouvelle-Calédonie, le paysage a-t-il fondé un sentiment d'appartenance collective ? Ou plutôt, comment a-t-il inspiré et mobilisé poétique et récits? L'activité minière laisse derrière elle désolation et stérilité : des montagnes saignées ou terrassées, des terres inexploitables. Le travail $\mathrm{y}$ est dangereux et humiliant, puisqu'il est rattaché à la condition pénale. Il y a donc une ambivalence dans l'évocation coloniale de ces paysages. Comment la mine peut-elle suggérer à la fois l'enfer et le rêve d'expansion coloniale? Cette perception ambiguë gagne en intensité si l'on se place du point de vue du colonisé où cet enfer, signifie l'arrachement aux terres ancestrales (Dauphiné, 1989), aux cultures, et aux clans. Bien que fondatrices de la société calédonienne, ces manières de percevoir le paysage minier ne sont pas figées et il convient d'en comprendre les transformations.

La littérature permet de saisir les complexités de la mémoire minière en Nouvelle-Calédonie. Mise en parallèle avec le politique, elle permet aussi de parler d'autonomie, d'indépendance, et même d'innovations technologiques ou d'écologie. Pourtant, ces projections contemporaines ne sont que l'une des nombreuses variantes des imaginaires miniers que la Nouvelle-Calédonie élabore depuis plus d'un siècle. Imaginaires qui ont pris forme à mesure que l'exploitation minière prenait de l'importance en Nouvelle-Calédonie depuis les années 1870. Quelle a été la première forme de cet imaginaire minier en NouvelleCalédonie ? Comment a-t-elle évolué ? Il s'agit de saisir comment le paysage minier se situe dans le fantasme "colonial" (rềve ou cauchemar) et comment il devient "familier", réel et peut mettre à l'épreuve le rêve colonial (Durand, 2008). C'est par les descriptions et évocations des paysages miniers offertes par la littérature que se donnent à voir les métamorphoses d'un imaginaire, donc celles d'une partie de la société. Nous parlons d'expression littéraire dans la mesure où c'est dans les chroniques de la presse coloniale que se retrouvent les premières traces 
du lyrisme minier. La première littérature, au sens de livres et d'auteurs qui exercent dans des genres (nouvelles, romans, etc.) où la mine devient un thème à part entière, apparaît avec les nouvelles que Georges Baudoux publie dans Le Messager à partir de 1898.

Il faut d'abord décrire ce qu'était l'idéologie coloniale en 1853, au moment de la prise de possession. L'État bonapartiste se devait d'éviter deux écueils pour faire accepter toute initiative de colonisation. En premier lieu, il s'agit d'éviter un dévoiement de l'idéologie coloniale française, fondée sur la "mission civilisatrice", et où la rencontre avec l' "Indigène » - vu comme barbare et plongé dans ses ténèbres - est également pensée comme une menace. Le second écueil était celui de la faillite économique, la colonisation s'accompagnant d'irrémédiables pertes financières dues aux coûts et aux incertitudes des expéditions et entreprises coloniales.

Napoléon III propage donc l'idée de "l'expansion outre-mer » selon laquelle travailler aux colonies enrichit la patrie et participe d'une certaine philosophie du progrès. Aussi est-ce une mission scientifique, celle de l'ingénieur Jules Garnier, qui va faire connaître la NouvelleCalédonie à Paris. Garnier avait pour tâche de faire l'inventaire des ressources de la jeune colonie ; l'idéal était d'y trouver de l'or. Menant sa mission de 1863 à 1866, il en tire un livre, Voyage à la Nouvelle-Calédonie, publié en 1868, qui sera un succès de librairie. Le texte se lit comme un récit de voyage, mais Garnier l'augmente de considérations scientifiques (géographie, géologie), de descriptions de paysages (il n'y a pas encore de mines), de tableaux de la vie des nouveaux colons français, d'observations ethnographiques et de récits d'aventure (batailles et scènes d'anthropophagie). Il s'agit de montrer que la France rayonne, que ses citoyens participent à diffuser son excellence (Seillan, 2008). Et comme dans tout rêve colonial, les paysages ne se regardent que pour ce qu'on peut en exploiter. Cette première mise en récit de la mine, et les autres qui se propagent au moins jusqu'en 1945 , sont des «discours d'appropriation de la terre" au sens où ils forment une partie de ce que Laurent Dubreuil définit comme « la phrase de possession" (Dubreuil, 2008: 78), que l'on peut définir comme les énoncés (Foucault 2008) qui, relayés par la presse et surtout la littérature, permettent d'assurer l'expansion coloniale, de faire entendre et appliquer la loi. Ici, la description des paysages dévastés est l'affirmation d'une appropriation (Le Meur, 2010) et constitue un motif de la "phrase de possession" (Dubreuil, 2008).
Du 18 avril $^{4}$ au 14 juin 1889, Francisque Ordinaire rédige dans Le Colon de la NouvelleCalédonie une chronique intitulée "De Paris à Nouméa", destinée à offrir un regard sur la métropole aux habitants de la colonie de Nouvelle-Calédonie 5 . Le développement de la SLN, fondée en 1880, est à l'époque la dernière grande épopée vécue par la colonie. La présence de Francisque Ordinaire dans les colonnes du Colon "fait entendre " la voix de la France, celle de la mère-patrie, aux colons ; et si possible sa fierté et son admiration pour sa colonie du Pacifique si longtemps discréditée par le bagne. En 1889, Ordinaire est en quête d'une nouvelle crédibilité politique. En effet, écarté de la politique depuis sa défaite aux élections législatives, il essaie de reconquérir un mandat politique. Son approche est plus stratégique qu'idéologique ; il tient à démontrer des aptitudes de gestionnaire. Avec l'essor du boulangisme (Winock, 1986 : 129-133), l'heure est à la formulation d'un nationalisme de gauche où montrer sa capacité à traiter des affaires coloniales peut constituer un argument électoral (Providence, 2005). Neuf ans après la fondation de la Société Le Nickel (SLN), Ordinaire veut donc rassurer, confirmer que l'exploitation du nickel fait la grandeur et l'utilité de la NouvelleCalédonie. Il associe informations historiques et informations économiques :

« Nous suivons toujours la rivière, dont nous ne cessons pas de traverser les zigzags et nous débouchons enfin vers une agglomération de quelques cases, qui forment le centre de Thio, où se trouvent les mines les plus importantes de la Société Le Nickel.

\section{Le Nickel}

Nous sommes dans le royaume du Nickel, de ce métal que l'industrie commence à employer pour les usages les plus divers, et pour lequel les gouvernements ont inventé la meilleure des réclames, en le mettant dans toutes les mains sous la forme de monnaie de billon.

Ce minerai fut découvert en Nouvelle-Calédonie dès 1863, par l'ingénieur des mines J. Garnier, mais les gisements ne furent déclarés qu'en 1873 sous le nom du "Mont-d'Or". Ils produisent environ cinq cents tonnes.

Mais la véritable exploitation sérieuse ne commença qu'en 1875 sur la "Boa-Kaine", appartenant à M. Hanckar, puis en même temps se fondait la compagnie le "Bel-Air" située à Houaillou. La production s'éleva à 10000 tonnes. " (Ordinaire, chronique du 22 avril 1889)

Francisque Ordinaire compose ici une nouvelle chronologie de la colonie, chronologie qui semble véritablement débuter avec l'exploitation du nickel. Surtout, il apporte de nouveaux

4. C’est la date la plus ancienne qui nous soit parvenue. Il semble que les débuts de la chronique soient antérieurs à cette date, mais les archives n'ont pas résisté aux ravages du temps.

5. Il en proposera également une version à Paris, dans l'édition illustrée du Figaro. 
personnages au récit de la colonisation: Jules Garnier, Hanckar et Higginson qui apparaissent comme les ingénieux héros d'une épopée. Cette mise en récit du monde minier semble avoir pour but de "créer", de fixer une image de la Nouvelle-Calédonie. Parlant du nickel, Francisque Ordinaire souligne qu'il est l'enjeu «de l'industrie » et " $[\mathrm{d}]$ es gouvernements " (ibid.). Ici, les chiffres dissimulent les paysages et confirment l'excellence coloniale. Dans sa chronique du 24 avril 1889, c'est à travers un inventaire de nations qu'il inscrit définitivement la NouvelleCalédonie sur la carte géopolitique mondiale. Elles font partie de celles qui ont choisi d'utiliser le nickel dans la fabrication de leur monnaie :

«On parle beaucoup du nickel et du projet de loi en préparation, qui aura pour but de transformer la monnaie de bronze en monnaie de nickel. Ce n'est pas une nouveauté, car les Américains ont créé cette monnaie en 1860 ; les Belges l'emploient depuis 1862, l'Allemagne depuis 1875 ; la Suisse l'a adoptée également.

On vient aussi d'accomplir cette réforme monétaire au Pérou, au Chili, au Brésil, en Colombie, à Honduras, et au Venezuela. En Europe, la Serbie a suivi cet exemple. " (Ordinaire, chronique du 24 avril 1889)

Le nickel néo-calédonien serait donc un élément incontournable de la révolution mondiale des pratiques monétaires : le nickel est "la monnaie " du Xx siècle imminent. Á travers l'inventaire des "nations du nickel " (ibid.), Ordinaire veut montrer que, grâce à la NouvelleCalédonie, la France peut intégrer cette élite.

Le 30 avril, il se prépareà l'ascension du Belvédère à Thio, pour y voir l'exploitation de la mine de nickel Santa-Maria, guidé par M. Bigillion, agent de la SLN, responsable de l'exploitation de cinq autres mines ${ }^{6}$ dans la région :

«En novembre 1887, le personnel du plateau du Belvédère se décompose ainsi : 112 blancs, sur lesquels 106 libérés, 70 Chinois [...] La Société emploie également des Canaques qui peuvent être considérés comme personnel du plateau ; 8 Néo-Hébridais font le service du dernier grand plan incliné et le minerai expédié ou mis en stock est manipulé par un blanc, et par 7 Chinois » (Ordinaire, 30 avril 1889).

Ordinaire rassure avec un exposé méthodique : la répartition raciale des tâches (où « le minerai expédié ou mis en stock est manipulé par un blanc»), des chiffres détaillés pour chacune des exploitations, autant de données sur «le personnel et son emploi » et " le prix de revient " qui confirment la réussite. Dans la chronique suivante, datée du 8 mai 1889 , il décrit une machinerie impressionnante qui rappelle à ses lecteurs les progrès de cette "fin de siècle industrielle » auxquels la France participe grâce à ses colonies. L'auteur décrit le ballet " [...] des chalands [qui] attendent la cargaison, qu'ils portent à bord des voiliers à destination d'Europe " (Ordinaire, 8 mai 1889). Il arrive ensuite sur le lieu de stationnement des machines :

"Après une heure de montée, nous arrivons au Belvédère, où se trouvent les engins nécessaires au fonctionnement du tramway et où aboutissent les différentes lignes qui conduisent aux diverses exploitations.

Nous continuons notre excursion sur un sol rocheux, métallique, du fer dont on peut ramasser les grains roulés, qui, mis dans un fusil bien ajusté, tueraient certainement les gracieuses hirondelles qui se balancent au-dessus de nos têtes, comme pour faire honte au génie humain de n'avoir pas encore trouvé la direction des ballons.

Le paysage est triste, avec les tons gris de ses arbustes qui ressemblent à des laurier-tins et la couleur rougeâtre du sol. Il y a là une mélancolie commune à tous les lieux où manquent l'eau et les grands arbres. " (ibid.)

Ce « paysage triste » contraste violemment avec la plupart des clichés "exotiques» de l'empire colonial. L'abondance de chiffres avancés par Ordinaire semble pouvoir les faire oublier. $\mathrm{Si}$ une image de la mine doit apparaître en arrièreplan, que ce soit celle d'une Europe mécanisée en marche vers le progrès.

Placée dès 1863 sous le signe de la colonisation pénale et, dès les années 1870 , sous celui de l'exploitation minière, sa colonisation fut ponctuée de déconvenues, de faillites, d'échecs agricoles, de récits des crimes commis par les évadés, et marquée par la grande révolte kanak de 1878. Un climat de peur et de tensions permanentes s'était installé, que la presse, en collaboration étroite avec l'administration coloniale, devait apaiser le plus souvent en rappelant la grande vocation française que tout colon devait respecter. Au moment de l'apparition des premières exploitations minières et surtout avec la fondation de la SLN (Société Le Nickel) en 1880, les paysages sont rapidement devenus un indispensable champ de projection et de controverses.

Ainsi, Ordinaire sera sévèrement critiqué en 1891 par Ernest Gegout (1854-1936) et Charles Malato (1857- 1938), deux déportés devenus anarchistes (Granier, 2008). Dans Prison fin de siècle, ils fustigent le déni de réalité d'Ordinaire et assimilent sa visite en Nouvelle-Calédonie à un exercice complaisant (Banaré, 2012 : 178190 ) et sa chronique, à un "compte-rendu de fonctionnaire " (Gegout et Malato, 1891: 166) superficiel. Cette critique de la chronique d'Ordinaire permet de mettre en évidence une 
tension entre les regards coloniaux posés sur les paysages miniers à cette période :

«[...] Le fonctionnaire inspecteur arrive à une date connue d'avance dans un milieu qu'il ignore complètement, festoye chez ceux dont il devrait éplucher la conduite, n'entend que les personnes qui peuvent accéder jusqu'à lui et ne voit que ce qu'on veut bien lui montrer. Finalement, il s'en retourne, au bout d'un temps très court, ayant composé son rapport de bribes officielles, ne connaissant rien du pays qu'il est censé avoir inspecté, et persuadé que tout y est pour le mieux. Toutes nos colonies sont des foyers d'intrigues et de tripotages, la Nouvelle-Calédonie peut-être plus qu'aucune autre, et dans ce pays où les classes dirigeantes affectent le plus profond mépris pour les transportés et les sauvages, tenus en quelques sortes en dehors de l'humanité, il se trouve que sauvages et transportés forment encore l'élément le moins corrompu " (ibid.).

On saisit ici tout ce qu'il y a de biaisé dans la chronique de 1889. Elle a été écrite par un politicien en campagne Charles Malato a été déporté en Nouvelle-Calédonie (Pisier, 1971) à l'âge de 17 ans avec ses parents communards ; c'est en regardant l'organisation de la vie de la colonie qu'il développe sa sensibilité anarchiste. Il quitte la Nouvelle-Calédonie en 1880, année de la fondation de la SLN, en ayant vécu la fièvre des premières spéculations minières ("ce foyer d'intrigues et de tripotages ") menées par John Higginson (Thompson, 2000). Constituée de "bribes officielles ", la chronique d'Ordinaire met en évidence un regard éloigné et un rapport aux paysages fortement travaillé par les rêves de prospérité coloniale. Ce regard a donné naissance à un des mythes fondateurs de la NouvelleCalédonie contemporaine: celui du pionnier, figure expiatoire qui a longtemps permis d'occulter les origines pénales (Barbançon, 2003). Dans Prison fin de siècle, Gegout et Malato montrent l'envers des paysages miniers mais le trait est peut-être exagéré. L'extraction du minerai et la transformation des paysages ne sont pas l'œuvre de travailleurs dévoués à la réussite coloniale, mais celle d'esclaves. "D'ailleurs, ajoutent-ils, en Nouvelle-Calédonie, l'esclavage est passé dans les mœurs et le mot "faire de la traite " est employé couramment " (Gegout et Malato, $1891: 169)$.

À partir de 1898, Georges Baudoux, élabore une rhétorique de la vocation coloniale (comme devoir patriotique); l'intention semble être d'apporter du pittoresque à une réalité. La différence est que Baudoux s'exprime à la fois en tant que colon et enfant du pays qu'il a fini par devenir (c'est le fils d'un fonctionnaire de l'administration pénitentiaire). Ses premières évocations du monde minier ont été des peintures sociales où apparaissait une communauté coloniale française idéalisée travailleuse et unie. Le monde de Baudoux est celui des "petits mineurs " qui fonctionne à une autre échelle que celui de la SLN ; les groupes y sont plus restreint et se côtoient plus facilement (Bencivengo, 1999). La "Chanson des cobaleurs" (Baudoux, 1978) composée vers 1895 fait de la mine le lieu de création d'un peuple qui chante en cœur, Baudoux célèbre la "famille des mineurs " liée par une solidarité virile. Surtout, il y retranscrit un argot particulier à la Nouvelle-Calédonie, celui des colons. Il esquisse un portrait de la colonie en société unie alors que la réalité présente des distinctions entre colons libres, bagnards, libérés ou évadés (Barbançon, 2003) qui régissaient les relations. Dans une autre nouvelle, "Clotho" (Baudoux, 1979), des bagnards aident la femme d'un propriétaire de mine à mettre au monde un enfant. C'est la rencontre entre les deux mondes coloniaux. Les bagnards, se retrouvent dans la demeure du propriétaire pour célébrer la naissance de l'enfant. Cette naissance devient ainsi le trait d'union de ce monde colonial brutal.

La description des paysages miniers paraît relever d'une expérience ambivalente. Baudoux semble adopter cette fois un point de vue autochtone à double sens, il y introduit la complexité des imaginaires d'une société en devenir. Dans "Sauvages et Civilisés " (Baudoux, 1979), écrit en 1915, il se livre à un exercice de subversion des regards et adopte, comme le titre peut le suggérer, un point de vue "Sauvage ". Le récit est le suivant: de jeunes colons rencontrent, pendant leur exploration d'un massif montagneux, Apollinaire ${ }^{7}$, un Kanak aux " théories d'un socialisme près de la nature " (Baudoux, 1979: 291). Il partage avec eux ses opinions sur la " civilisation" :

«Votre civilisation à vous est si fausse que, sans vouloir en convenir, vous la fuyez » (Baudoux, 1979 : 292).

Le narrateur entame alors une méditation sur sa position en tant que colon et devient réceptif à ces paysages. Il écoute les paroles rivales des

7. Rien n'en atteste la lecture chez Baudoux, mais il est tentant de voir dans ce personnage une évocation du surréaliste Guillaume Apollinaire. Les dates concordent et, comme l'a montré Bernard Gasser dans son étude sur Baudoux, ce dernier animait activement la vie littéraire locale; les libraires de Nouméa étaient régulièrement informées des avant-gardes parisiennes. Le Messager, journal littéraire tenu par Alain Laubreaux, servait, en effet, de relais entre Paris et Nouméa. Il est donc fort probable que des exemplaires d'Alcools ont été vendus en Nouvelle-Calédonie. Ayant publié son recueil Alcools deux ans avant la parution de "Sauvages et Civilisés ", Apollinaire exprimait déjà, dans les vers du poème "Zone », un malaise européen. Il en avait " assez de vivre dans l'antiquité grecque et romaine » et évoquait un âge premier idéalisé aux antipodes parmi les "fétiches d'Océanie et de Guinée [...] Christs inférieurs des obscurs espérances »; nous sommes ici proches du « socialisme près de la nature " du personnage de la nouvelle de 1915. 

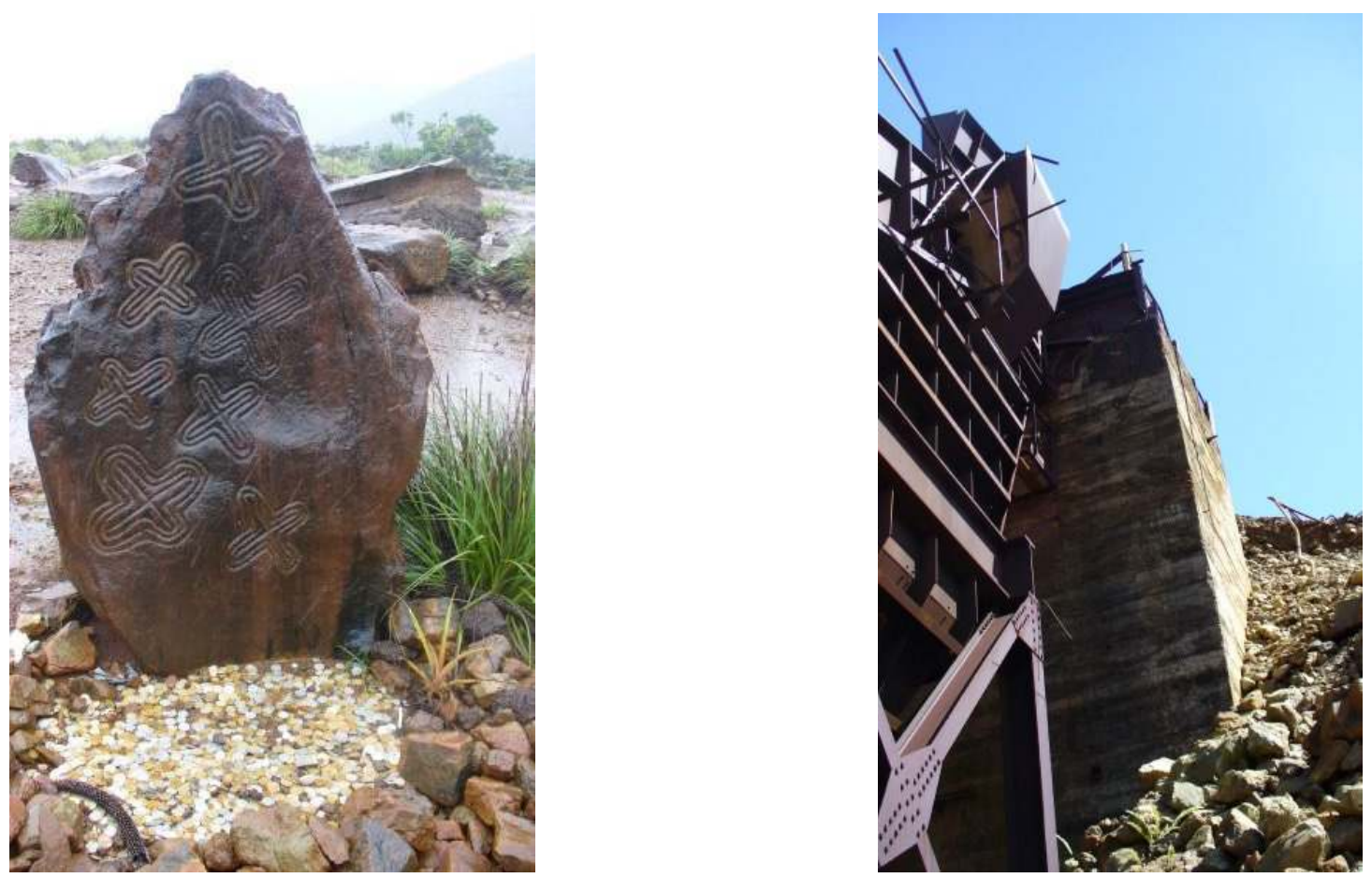

Figures 1-2. - Pétroglyphes et infrastructures sur le site minier abandonné de Kaa Wi Paa (Kouaoua, Nouvelle-Calédonie) illustrant la dualité mémorielle (2008, cliché Eddy Banaré)

" chaînes sombres (et des) petites montagnes " (Baudoux, 1979 : 302). Les "chaînes " qui abritent les filons sont les premières à se faire entendre :

"Elles semblaient, ces chaînes majestueuses, dire avec orgueil aux plus petites qu'elles :

Reculez-vous! Faites-nous place! Nous sommes les serpentines, l'épine dorsale de la Calédonie. C'est nous qui faisons la loi, nous donnons le mouvement, nous créons l'activité. Dans notre sein nous renfermons les laves infernales de Pluton, cristallisées en des richesses inépuisables.

Voyez ces plaies jaunes, béantes, qui s'ouvrent par gradins dans nos larges poitrines; elles y sont creusées pour en extraire le nickel qui est notre chair.

Et ces entrailles profondes, sanglantes, qui bâillent dans notre derme d'argile rouge ; elles ont été incisées pour arracher nos nervures de chrome qui vont, par le monde, durcir les métaux.

Regardez ces trous noirs qui pénètrent dans nos entrailles, ainsi que des antres de cyclopes; ce sont des tunnels obscurs et tortueux qui vont dans des gîtes pleins de mystères saigner nos veines bleuies de cobalt » (Baudoux, 1979 : 302).

"Le nickel qui est notre chair»: la "richesse et [la] puissance " que vantent ces montagnes disent également une violence faite à la terre. Extraire le minerais, c'est faire une saignée, c'est profaner et appauvrir la terre. Les montagnes avertissent: "dans notre sein nous renfermons les laves infernales de Pluton, cristallisées en des richesses inépuisables »(Baudoux, 1979 : 303). Le stockman éprouve les contradictions du colon fier de la réussite annoncée par les prospects, mais attaché à des paysages qui sont désormais pour lui, puissamment évocateurs. La réponse des "petites montagnes " est un avertissement concernant le futur. Le verbe de Baudoux prend des accents écologiques :

"Nos allées arrosées et fertiles, nos alluvions chargés d'humus qui comblent les estuaires de nos rivières, nos collines couvertes de sillons étagés, font vivre depuis des siècles des générations d'hommes noirs. Nous sommes là, attendant la houe et la charrue. Aux plantations de café, de coton, de maïs, à toutes les cultures, nous offrons nos terrains généreux ; il suffit de savoir nous choisir. Sur notre littoral, et sur les berges de nos rivières, les cocotiers poussent avec vigueur, par leurs racines avides, ils s'imprègnent des eaux de la mer. Respectez-nous ! Nous sommes l'agriculture " (Baudoux, 1979 : 302-303).

Un dilemme insoluble apparaît dans l'esprit du colon entre sa vocation première, celle des prospects qui faisaient sa fierté, et une expérience intime des paysages. Déjà, en formulant la critique de la " civilisation " par la voix du "Sauvage ", Baudoux reconnait le monde colonisé qu'il présente comme porteur de vérités essentielles. Mais, en mentionnant les "générations d'hommes noirs" (ibid.) qui ont cultivé les flancs des montagnes, il renverse la doxa coloniale raciste sur l'absence de profondeur historique. Aussi, par le jeu de la prosopopée, il y a également la reconnaissance d'un savoir qui ne pouvait être possible quà partir d'un certain niveau de connivence, en bref, 
d'une rencontre. L'harmonie, la connaissance de la terre et de la nature reconnue au monde kanak est une rupture dans la "phrase de possession" (Dubreuil, 2008) également fondatrice d'une mémoire. Lauteur formule les termes d'une reconnaissance nouvelle; celle de l'autre qu'il était, selon le mot d'ordre colonial, nécessaire de "civiliser». Surtout, celui qui apparait en filigrane du texte de Baudoux est désormais un enfant de cette terre :

«Plus tard! Vous, mines gonflées de minerais et d'orgueil, quand vous aurez prodigué toutes vos richesses aux spéculateurs étrangers, vous ne laisserez après vous que des ruines; vos terres fouillées, vidées de leurs trésors, resteront éternellement stériles. Vos montagnes déchiquetées, arides comme des paysages lunaires, seront l'image de la désolation et de la mort.

Alors que nous! Nous les petites montagnes, les dédaignées de l'heure présente, nous serons toujours là, verdoyantes, grasses, riches, fécondées par le travail, par le labeur persévérant des petits. À ceux qui auront eu confiance en nous, nous donnerons l'abondance et le bien-être, car nous sommes la terre qui nourrit, la terre à laquelle on s'enracine. Nous sommes l'agriculture, nous sommes l'avenir qui crée pour lui des œuvres durables. Et vous, mines gaspilleuses, vous êtes le présent qui butine, s'envole et ne laisse rien.

Ainsi parlaient les montagnes. Nous comprenions leur éloquent langage accompagné de démonstrations panoramiques; mais nous ne voulions pas nous immiscer dans leurs affaires. »(Baudoux, 1979 : 303)

Sa pensée, encore imprégnée par l'idéal civilisateur, est pourtant perméable à une altérité qui joue ici le rôle d'un contradicteur tragique : " vos montagnes déchiquetées, arides comme des paysages lunaires, seront l'image de la désolation et de la mort" (ibid.). Baudoux sème ainsi le trouble en formulant le vœu d'une colonie tournée vers l'agriculture à rebours des rêves pionniers de la dernière moitié du $\mathrm{XIX}^{\mathrm{e}}$ siècle. Cette nouvelle met en évidence ce que Homi K. Bhabha appelle "l'absurdité coloniale" (Bhabha, 2007 : 201) au sein de laquelle « [...] les dualités qui divisent traditionnellement l'espace colonial : nature/culture, chaos/civilité " (ibid.) sont remises en cause.

Le succès de Baudoux était, en effet, fondé sur la connaissance du monde kanak. En tant que stockman, il se vantait d'avoir pu assister aux veillées de contes autour du feu, d'avoir reçu un nom kanak, "Thiosse ". Ceci lui confere une certaine crédibilité, et la société coloniale lui reconnait une expérience. En effet, le paradoxe de Baudoux est d'être profondément attaché à une terre; sentiment qui ne peut être compris qu'en adoptant un regard autre, celui de ceux que la mission coloniale ordonne de dominer. Baudoux ne peut donc formuler que deux possibilités : céder aux impératifs coloniaux et industriels ou préférer « les œuvres durables » de « l'agriculture ».
Les paysages miniers de la Nouvelle-Calédonie soumettaient donc les auteurs (également idéologues de l'empire) à un impossible esthétique. La désolation, la violence des populations qui y travaillent (condamnés et ouvriers sous contrat de "chair humaine") en faisaient un enfer où il semble impossible de chanter la grandeur coloniale. La mine ne peut donc se rêver que « de loin ", en périphérie ou, comme Ordinaire en 1889, en suggérant le courage nécessaire pour y travailler à travers des descriptions paysagères. En 1915, Baudoux tente d'affronter cet impossible, mais toujours en périphérie. Du point de vue de la temporalité de "Sauvages et Civilisés », la mine n'existe pas encore puisque nous y suivons des prospecteurs. En revanche, elle est évoquée comme une catastrophe à venir. Les ravages sur les flancs des montagnes annoncent uniquement l'essor économique et les progrès techniques ; l'agriculture est, en revanche, le rêve d'une harmonie retrouvée.

\section{Ouvertures et interrogations kanak}

Il en va tout autrement pour le monde kanak, où l'activité minière suggère la variété de la domination coloniale: spoliations foncières, déportation, profanation des lieux de culture et, plus encore, des lieux tabous. Il faut, cependant, rappeler l'impact intellectuel de Jean-Marie Tjibaou, qui a participé à réinventer l'imaginaire de l'activité minière en l'intégrant à sa vision de l'indépendance et en affirmant la possibilité et la nécessité pour les Kanak d'y prendre part (Tjibaou, 1996 ; voir aussi Mokaddem, 2011). C'est surement dans le discours qu'il prononce en 1978 pour le Congrès de l'Union Calédonienne (UC) à Maré que l'on assiste à un changement de perspective. Dans le prolongement du mot d'ordre d'alors - "Calédonie, notre patrimoine " - Jean-Marie Tjibaou affirme que la mine est un "patrimoine" (Mokaddem, 2009) au même titre que les chants, les contes ou les pratiques de pêche et de chasse. Le défi était lancé de transformer l'industrie minière pour la faire entrer dans une réalité kanak.

Dans l'expression et l'histoire littéraires de la Nouvelle-Calédonie, l'activité minière a donc successivement été formulée comme un projet puis comme un haut fait de la conquête coloniale. Projet, dans la mesure où il s'agissait, dès 1853 , de trouver la vocation économique de la colonie. Conquête, car une fois entamée à partir de la fin des années 1870, l'exploitation du nickel devenait fondatrice de la Nouvelle-Calédonie.

On peut dire que, jusqu'aux débuts des années 1970 (à la période du «boom» du nickel), la représentation proposée par Baudoux va connaître une cristallisation en même temps qu'un début de remise en question par le monde 
kanak. La première interférence vient des Foulards rouges et du Groupe 78, entités politiques issues de la première génération d'étudiants kanak influencés par Mai 68 et les lectures de Fanon (Chappell, 2003/2013). Les montagnes éventrées et les plateaux miniers ne sont pour eux que les symboles d'un vieil héritage colonial, de terres confisquées et d'inégalités sociales rampantes. À défaut de faire disparaître la mine, peut-être faut-il la conquérir, la transformer en la plaçant au cour d'un nouveau projet politique et économique.

Sur le plan littéraire, les œuvres de Déwé Görödé et Pierre Gope procèdent à un inventaire historique des douleurs, leur écriture relève de ce que Patrick Sultan nomme le « trauma », c'est-à-dire :

«[qu’elles] sont des réponses (qui peuvent être multivoques, complexes et obliques) à un passé colonial qui a laissé des traces qu'elles explorent et d'une mémoire qu'elles ravivent " (Sultan, $2011: 28$ ).

Ici, les paysages sont évoqués à partir d'un vécu colonial de la mine : celui des terres profanées, des clans déportés et démantelés. Aussi, lorsque Déwé Görödé publie le recueil Sous les cendres des conques en 1985, on ne peut que mieux saisir ce qui a été un des points de départ d'un nouveau regard posé sur le nickel en Nouvelle-Calédonie, l'amorce d'un changement d'imaginaire. La section $3 \mathrm{du}$ recueil, intitulée "Terre Nickel", réunit onze poèmes composés entre 1970 et 1985, certains alors que Déwé Görödé était incarcérée pour son activisme politique. Ces poèmes affirment ce que la mine ne doit plus être ; en montrant une réalité, ils appellent à en inventer une autre. C'est la première interférence avec le mythe colonial élaboré depuis les débuts de la prospection. Voici ce qu'en dit Déwé Görödé dans son poème «Et les prospectus » :

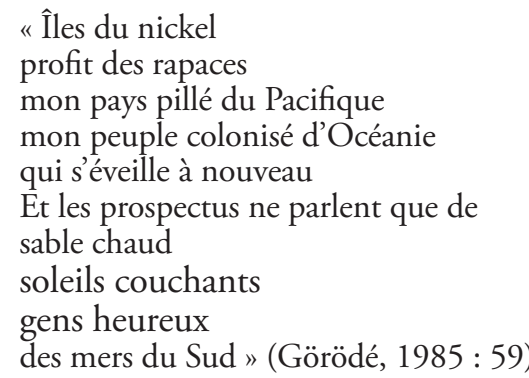

Les « ̂̂les du nickel » relèvent d'une propagande coloniale. Dans Sous les cendres des conques, la mine c'est aussi «Madame Multinationale [qui] a des tentacules intercontinentales [et] ne comprend aucune langue de la terre » Görödé, 1985 : 6364). Dans ce poème daté de 1970, l'aventure et le labeur des stockmen et prospecteurs ont disparu, ils ont désormais leur envers, qui est associé aux mensonges touristiques des "prospectus " : ceux qui attirent les spéculateurs "rapaces". Et les images peuvent se faire encore plus violentes :

\section{« Kanaky}

quelque part en toi brille le nickel

qu'ils voudraient tous laper de leur museau

de toutous des multinationales. " (Görödé, 1985: 63-64)

Nommer le pays rêvé (Darot, 1997), c'est déjà en reprendre possession, l'inventer et lui donner vie. Cependant, la mine que regarde Déwé Görödé tient encore à distance le monde kanak. Les différentes séquences politiques que la Nouvelle-Calédonie connait de 1984 à 1988 contribueront à un rapprochement. La poésie permet de s'emparer des mots " nickel », " mine " ou du sigle de la «SLN" et de réveiller une autre mémoire. En 1985, dans le poème " Nickel », ils renvoient à la désolation : "les eaux jaunâtres ", "les crêtes déchirées et les pentes déchiquetées" prises d'assaut par des "tentacules de fer». Quant à la SLN, elle représente "trois lettres incandescentes dans la chair les entrailles du pays " (ibid.). Le titre du poème "Fer rouge", renvoie au marquage, à l'image des corps suppliciés. Le paysage qui se dessine en négatif est celui du silence et de la mort. Surtout, son évocation permet ici de formuler un projet tout aussi poétique que politique. Le nom de "Kanaky " est en effet prononcé ; la désolation des paysages annonce ici un pays à naître et permet de proclamer une différenciation.

L'accord de Nouméa en 1998 annonce la nécessité de nouvelles symboliques où les intérêts miniers doivent être conjugués avec d'autres valeurs. Comment entrer dans la mine quand on est kanak? Comment regarder une industrie minière qui a d'abord signifié spoliation et humiliation ? Ne court-on pas le risque d'une corruption, d'une trahison des siens? Le monde kanak qui aborde la question minière trois ans après la signature de l'accord de Nouméa est travaillé par des dynamiques douloureuses et contradictoires. Dans Le dernier crépuscule (2001), ces questions mettent en tension une tribu isolée de la chaîne; Pierre Gope met en scène les négociations qui ont préfiguré la naissance du projet Koniambo (Horowitz, 2003, 2004). La montagne est le premier paysage de la pièce - le décor - lieu de mémoire, lieu des ancêtres. La mine apparait alors en négatif. Elle est d'abord une rumeur venue de la ville. Cette rumeur grandit :

«Parait-il que notre village sera renversé, et que nous allons vivre à l'envers »(Gope, 2001 : 17)

demande le Vieux Saké. Un jeune Kanak diplômé "Chargé de mission" (Gope, 2001: $8)$, représentant de la compagnie minière, tente 
de convaincre les anciens et les autres "jeunes" en brandissant des mots - "modernité", "développement ", "technologie " - que Gope fait apparaitre comme de véritables mirages, de nouvelles séparations entreles générations. Pour une partie des anciens, la mine est l'imminence d'une catastrophe; pour les jeunes, elle représente un nouvel horizon : la technologie, le développement, l'occasion de participer au monde. Le constat du Vieux Wapo est plein de dépit :

"Aujourd'hui, c'est par la bouche d'un enfant du pays que l'État parle, pour nous demander de partir et d'abandonner nos terres et nos dieux " (Gope, $2001: 32$ ).

Mais l' " enfant du pays ", après avoir obtenu l'accord des anciens, décède brutalement. Et les questions demeurent, ainsi que les spéculations les plus alarmantes. Cette mort est vécue comme une fatalité, le prix à payer d'une compromission, Pa Saké tranche: "la technologie, c'est celle qui a tué notre frère, c'est celle qui ronge nos montagnes, comme des bancoules dans les bancouliers " (Gope, 2001 : 75). La démarche initiée par le jeune Kanak diplômé est assimilée à une trahison, symboliquement, il est " mort " aux yeux des anciens en voulant livrer la terre ancestrale aux machines. Ce à quoi un "Jeune " réplique: "la technologie, c'est une nécessité aujourd'hui " (ibid). Naïveté, lucidité implacable ou pire, corruption? Ici, la mine nous confronte à un irrésolu, en remettant en question le lien à la terre qui est au coeur du monde kanak (Horowitz, 2008).

Le dernier crépuscule (2001) est publié un an après le décès tragique de Raphael Pidjot - la référence est à peine dissimulée -, premier Kanak devenu président de la sMSP (Société minière du Sud Pacifique) en 1990, soit deux ans après la signature des accords de Matignon (Pitoiset et Wéry, 2009). Les questions soulevées par le drame de Pierre Gope dépassent l'aspect littéraire de la représentation minière. Elles mettent en évidence un clivage entre tenants de la construction économique de l'indépendance et tenants d'une préservation écologique de la nature en lien avec la société kanak. Ce débat interne au monde politique kanak se perpétue aujourd'hui par le recours par le comité Rhéébù Nùù crée en 2002 au discours de l'autochtonie sur la légitimité (Horowitz, 2008).

\section{Conclusion}

C'est l'expression d'une confrontation des imaginaires et celle du défi de l'adaptation qui se pose à travers la littérature. Un nouvel horizon de possibles a été déployé, reste à savoir comment les investir (Bensa et Freyss, 1994) dans le cadre de l'émergence du nationalisme kanak et la signature des accords politiques (Matignon en 1988 et Nouméa en 1998). La mine est devenue une présence quotidienne dont on fabrique en permanence de nouvelles représentations, processus auquel l'expression littéraire participe activement. Elle constitue un champ de projections à partir duquel l'espace, l'histoire et les rapports sociaux peuvent être pensés et sont à même d'évoluer (Demmer, 2007). La signature de l'accord de Nouméa en 1998 a mis en lumière les enjeux déployés autour des revendications indépendantistes kanak et l'élaboration d'un imaginaire de l'exploitation minière où se superposent pragmatisme économique, désir d'indépendance, reconnaissance culturelle, souci de paix civile et écologie.

Ainsi, les expériences du nickel restituées par la littérature donnent à voir une complexité anthropologique à valoriser dans ce "fairemémoire " que préconise l'accord de Nouméa. On peut considérer que l'écrivain puise dans un terreau particulier et que son œuvre n'est qu'un écho amplifié de rêves, de projets, d'utopies, de joies ou de haines. La littérature, lorsqu'elle s'empare de la mine, souligne la plupart des contradictions, des désirs d'invention, de contestations qui travaillent et font la NouvelleCalédonie contemporaine.

\section{BIBLIOGRAPHIE}

AcCord sur la Nouvelle-Calédonie signé À Nouméa le 5 MaI 1998, 1998 (27 mai). Journal officiel de la République française (JORF) 121, p. 8039.

Anderson Benedict, 2006 (1éd 1983). L'imaginaire national: Réflexions sur l'origine et l'essor du nationalisme, Paris, La Découverte.

Anova-Ataba Apollinaire, 1969. Deux exemples de réflexions mélanésiennes, Journal de la Société des Océanistes 25, pp 201-237.

Banaré Eddy, 2012. Les récits du nickel en Nouvelle-Calédonie (1853-1960), Paris, Honoré Champion, Collection Francophonies.

Barbançon Louis-José, 2003. L'archipel des forçats. Histoire du bagne de Nouvelle-Calédonie (1863-1931), Paris, Presses universitaires du Septentrion.

Baudoux Georges, 1979. Les blancs sont venus, t. 2, Nouméa, Société d'études historiques de la Nouvelle-Calédonie, Publications de la SEHNC 20.

Bencivengo Yann, 1999. La mine en NouvelleCalédonie, Nouméa, Île de lumière. 
-, 2008. La mine, conflits d'hier et d'aujourd'hui, in Sylvette Boubin-Boyer (éd.) Conflits et Guerres mondiales en Nouvelle-Calédonie et dans sa région (t. I), Paris, L'Harmattan, pp 287-301.

Bensa Alban et Jean Freyss, 1994. La société kanak est-elle soluble dans l'argent...? Terrain 23 , pp. 11-26.

Bнавна Homi K., 2007 (1éd 1994). Les lieux de la culture, Paris, Payot.

Buttet Catherine, 2000. La banque de la Nouvelle-Calédonie. Existence éphémère, expérience oubliée (1874-1877), Cahiers d'Histoire 45, 1, pp 71-105.

Chappell David, 2003. The Kanak Awakening of 1969-1976: Radicalizing Anti-Colonialism in New Caledonia, Journal de la Société des Océanistes 117 : Nouvelle-Calédonie. 150 ans après la prise de possession, Isabelle Leblic (éd.), pp. 187-202 (http://jso.revues. org/1268).

-, 2013. The Kanak Awakening: The Rise of Nationalism in New Caledonia, Honolulu, University of Hawai'I Press, Pacific Island Monograph Series, 27.

Collot Michel. 2011. Pour une géographie littéraire, Fabula-LhT $8:$ Le partage des disciplines (http://www.fabula.org/lht/8/collot.html).

Coquilhat Georges, 1987. La Presse en Nouvelle-Calédonie au XIX siècle, Nouméa, Société d'études historiques de la Nouvelle-Calédonie, Publications de la SEHNC 38.

Darot Mireille, 1997. Kanaky ou Caillou? Implicites identitaires dans la désignation de la Nouvelle-Calédonie, Mots 53: La Nouvelle-Calédonie après les accords de Matignon, pp. 8-25 (http://www.persee.fr/web/ revues/home/prescript/article/mots_02436450_1997_num_53_1_2444).

DAuphiné Joël, 1989. Les spoliations foncières en Nouvelle-Calédonie (1853-1913), Paris, L'Harmattan.

Demmer Christine, 2007. Autochtonie, nickel et environnement : une nouvelle stratégie kanake, Multitudes 39, pp. 43-48 (http://www. vacarme.org/article1306.html).

Didi-Huberman Georges, 2010. Remontages du temps subi : L'œil de l'histoire 2, Paris, Les Éditions de Minuit, Paradoxe.

Do Tess, 2008. Exile: Rupture and Continuity in Jean Vanmai's Chân Dang and Fils de Chân Dang, in Paul Allatson et Jo McCormack (éd), Exiles Cultures, Misplaced Identities, Rodopi, pp. 151-172.
Dubreuil Laurent, 2008. L'empire du langage, colonies et francophonie, Paris, Hermann, Savoirs Lettres.

Durand Jean-François, 2008. Littératures coloniales, littératures d'empire ?, Romantisme 139, 1, pp. 47-58 (http://www.cairn.info/ revue-romantisme-2008-1-page-47.htm).

Foucault Michel, 2008 (1 $1^{\mathrm{e}}$ éd. 1969). L'archéologie du savoir, Paris, Gallimard, Tel.

Gagné Natacha et Marie SALAün, 2013. Les chemins de la décolonisation aujourd'hui : perspectives du Pacifique insulaire, Critique Internationale 60, pp. 112-132.

GARDE François, 2005. Le préambule de l'accord de Nouméa, prologue d'une histoire officielle ?, Revue française de droit constitutionnel 64, pp. 805-811.

Garnier Jules, 1991 (1 $1^{\mathrm{e}}$ éd. 1867). Voyage à la Nouvelle-Calédonie 1863-1865, Paris, Zulma.

Gasser Bernard et Alain Sollier, 1996. Georges Baudoux, la quête de la vérité, Nouméa, Grain de Sable.

Gegout Ernest et Charles Malato, 1891. Prison fin de siècle : souvenirs de Pélagie, Paris, G. Charpentier et E. Fasquelle.

Gope Pierre, 2001. Le dernier crépuscule, Nouméa, Grain de Sable.

GöröDÉ Déwé, 1985. Sous les cendres des conques, Nouméa, Édipop.

Granier Caroline, 2008. Les briseurs de formules. Les écrivains anarchistes en France à la fin du XIX siècle, Cœuvres-et-Valsery, Ressouvenances.

Horowitz Leah S., 2003. La micropolitique de la mine en Nouvelle-Calédonie: analyse des conflits autour d'un projet minier au sein d'une communauté kanak, Journal de la Société des Océanistes 117 : Nouvelle-Calédonie. 150 ans après la prise de possession, Isabelle Leblic (éd), pp. 254-271.

—, 2004. Toward a Viable Independence? The Koniambo Project and the Political Economy of Mining in New Caledonia, The Contemporary Pacific 16, 2, pp. 287-319.

—, 2008. Destroying God's creation or using what He provided?: Cultural models of a mining project in New Caledonia, Human Organization 67, 3, pp. 292-306.

JAMESON Fredric, 2012 (1éd. 1981). Linconscient politique : le récit comme acte socialement politique, Paris, Questions théoriques.

Le Meur Pierre-Yves, 2009. Opérateurs miniers, gouvernementalité et politique des ressources 
à Thio, Nouvelle-Calédonie, Actes du Pacific Science Intercongress (Papeete, 3-6 mars 2009) (http://webistem.com/psi2009/output_directory/cd1/Data/articles/000166.pdf).

-, 2010. La terre en Nouvelle-Calédonie: pollution, appartenance et propriété intellectuelle, Multitudes 41, pp. 91-98.

Merle Isabelle, 1995. Expériences coloniales, la Nouvelle-Calédonie (1853-1920), Paris, Belin.

-, 1998. La Construction d'un droit foncier colonial. De la propriété collective à la constitution des réserves en Nouvelle-Calédonie, Enquête 7, pp. 97-126.

Mokaddem Hamid, 2007. Euvres et trajectoires d'écrivains de Nouvelle-Calédonie, Nouméa, Expression - Province Nord.

—, 2009. Pratique et théorie kanak de la souveraineté... 30 janvier 1936, Jean-Marie Tjibaou, 4 mai 1989..., Nouvelle-Calédonie, province Nord.

—, 2011. Le discours politique kanak. Jean-Marie Tjibaou, Rock Déo Pidjot, Éloi Machoro, Raphaël Pidjot, Koné, Les Éditions de la province Nord.

-, 2014. Apollinaire Anova. Une conception kanak du monde et de l'histoire (1929-1966), Nouméa/Marseille, Expressions, La courte échelle/Éditions transit.

Ordinaire Francisque, 1889. Chroniques (22 avril 1889, 24 avril 1889, 30 avril 1889, 8 mai 1889), Le Colon de la Nouvelle-Calédonie, archives de la Nouvelle-Calédonie.

Petit Michèle, 2010. " Ici, y’a rien ! " La littérature, partie intégrante de l'art d'habiter, Communications 87 : Autour du lieu, pp. 65-75.

Pisier Georges, 1971. Les déportés de la Commune à l'île des Pins, Nouvelle-Calédonie, 1872-1880, Journal de la Société des océanistes
31, pp. 103-140 (http://www.persee.fr/web/ revues/home/prescript/article/jso_0300953x_1971_num_27_31_2322).

Pitoiset Anne et Claudine Wéry, 2009. Mystère Dang, Paris, Rayon Vert.

Providence Mathieu, 2005. Boulanger avant le boulangisme : un officier colonial tombé en République, Politix 72, 4, pp. 155-179.

Rancière Jacques, 2010. La parole muette. Essai sur les contradictions de la littérature, Paris, Hachette Littérature, Collection Pluriel.

Ricceur Paul, 1985. Temps et récit (t. III), Paris, Seuil, Points Essais.

Roe Emery, 1994. Narrative Policy Analysis. Theory and Practice, Durham-London, Duke University Press.

SeILlan Jean-Marie, 2008. La (para)littérature (pré) coloniale à la fin du XIx ${ }^{e}$ siècle, Romantisme 139, 1, pp. 33-45 (http://www.cairn. info/revue-romantisme-2008-1-page-33. htm).

Sultan Patrick, 2011. La scène littéraire postcoloniale, Paris, Le Manuscrit, Esprit des Lettres.

Thompson Anne-Gabrielle, 2000. John Higginson. Un spéculateur-aventurier à l'assaut du Pacifique: Nouvelle-Calédonie, Nouvelles-Hébrides, Paris, L'Harmattan.

Tuibaou Jean-Marie, 1996. Présence kanak, Paris, Odile Jacob.

Vanmaï Jean, 1980. Chân Dang: Les Tonkinois de Calédonie au temps colonial, Nouméa, Société d'Études historiques, Publication de la SEHNC 24.

—, 1983. Fils de Chân Dang. Nouméa, Éditions de l'Océanie.

Winock Michel, 1986. La fièvre hexagonale: les grandes crises politiques, 1871-1968, Paris, Seuil, Point Histoire. 